Torres Gordillo, Juan Jesús; Perera Rodríguez, Víctor Hugo; Cobos Sanchiz, David (2014). Investigación evaluativā de la formación online en prevención de riesgos laborales y medio ambiente: un análisis cualitativo de los programas impartidos por la fundación istas. Revista de Investigación Educativa, 32 (1), 73-90.

http://dx.doi.org/10.6018/rie.32.1.166621

\title{
INVESTIGACIÓN EVALUATIVA DE LA FORMACIÓN ONLINE EN PREVENCIÓN DE RIESGOS LABORALES Y MEDIO AMBIENTE: UN ANÁLISIS CUALITATIVO DE LOS PROGRAMAS IMPARTIDOS POR LA FUNDACIÓN ISTAS
}

\author{
Juan Jesús Torres Gordillo(l),Víctor Hugo Perera Rodríguez ${ }^{(1)}$ y David Cobos Sanchiz ${ }^{(2)}$ \\ (1) Universidad de Sevilla \\ (2) Universidad Pablo de Olavide
}

\section{RESUMEN}

Este artículo presenta los resultados de una investigación evaluativa de la formación online impartida por el Instituto Sindical de Trabajo, Ambiente y Salud (ISTAS) sobre prevención de riesgos laborales y medio ambiente. Se pretende conocer el impacto real en el desempeño laboral y la satisfacción de los participantes sobre la formación recibida. La muestra se centra en cinco cursos de formación, con un total de 184 participantes. Se utiliza la entrevista estructurada online para la recogida de datos. El método de Análisis de Contenido permite aplicar un sistema de categorías que define operativamente los procesos de evaluación de la satisfacción en la formación online. Los resultados indican una alta satisfacción de los participantes y un impacto medio en la práctica real. Se concluye con una serie de propuestas que pueden ayudar a mejorar en el futuro los cursos analizados y otras acciones similares impartidas mediante esta modalidad de formación.

Palabras clave: investigación evaluativa; satisfacción; impacto; salud laboral.

\footnotetext{
Correspondencia:

Juan Jesús Torres Gordillo. Dpto. Métodos de Investigación y Diagnóstico en Educación. Universidad de Sevilla E-mail: juanj@us.es

Víctor Hugo Perera Rodríguez. Dpto. Didáctica y Organización Educativa. Universidad de Sevilla

E-mail: vhperera@us.es

David Cobos Sanchiz. Dpto. Ciencias Sociales. Universidad Pablo de Olavide

E-mail: dcobos@upo.es
} 


\title{
EVALUATIVE RESEARCH OF E-LEARNING IN ENVIRONMENT AND OCCUPATIONAL SAFETY:A QUALITATIVE ANALYSIS OF THE PROGRAMS OFFERED BYTHE ISTAS FOUNDATION
}

\begin{abstract}
This article presents the results of an evaluative research study of e-learning in environment and occupational safety offered by the Labor, Environment and Health Union Institute (ISTAS). The objective of this study was to explore the real impact on labor activity and participants' satisfaction. The sample was made up of 184 participants from five training courses. An online structured interview was used for collecting data. Content Analysis method enabled the use of a system of categories, which defined the process of evaluating satisfaction with e-learning. The results showed participants' high satisfaction and a moderate impact. A number of proposals for the improvement of courses and similar online actions are presented.

Keywords: evaluative research; satisfaction; impact; occupational health.
\end{abstract}

\section{INTRODUCCIÓN}

Aunque la utilización de los numerosos recursos y posibilidades, que por entonces ya ofrecía Internet, significó en los años noventa una auténtica revolución de los enfoques tradicionales de la enseñanza a distancia, posteriormente factores como la fuerte demanda social de educación y formación continua o el enorme desarrollo de las tecnología han venido a potenciarla sobremanera. Ya en 2004 el e-Learning, incluyendo la formación blended-learning o semipresencial, movía en nuestro país unos cuarenta millones de euros y era utilizado por el $40 \%$ de las grandes empresas (Doxa, 2005). Esta modalidad de enseñanza y formación ha ido expandiéndose por su adaptación a las necesidades y posibilidades de las personas adultas, porque ofrece la posibilidad de que puedan aprender sin necesidad de abandonar su puesto de trabajo o bien de dedicar largas horas a sesiones de formación presenciales. Ha permitido que personas que viven en zonas alejadas de las capitales puedan formarse. Igualmente facilita que otras personas con movilidad limitada o bien con responsabilidades de atención a menores, mayores o dependientes puedan también participar de un aprendizaje activo (Marcelo, 2011).

En la formación de adultos, especialmente en lo que se refiere a la formación ocupacional y continua, el e-Learning posibilita el aprendizaje de un buen número de contenidos y el mejoramiento de numerosas habilidades. Carretero (2006) señala, entre ellas, la formación en Tecnologías de la Información y la Comunicación (TIC), el conocimiento del funcionamiento de software, los idiomas, el perfeccionamiento profesional y la seguridad e higiene. A estos últimos temas, la seguridad e higiene en el trabajo, la prevención de riesgos y el medio ambiente de trabajo, dedica la Fundación Instituto Sindical de Trabajo, Ambiente y Salud (ISTAS) buena parte de sus esfuerzos. ISTAS es una fundación autónoma que tiene el objetivo general de impulsar actividades de progreso social para la mejora de las condiciones de trabajo, la protección del medio ambiente y la promoción de la salud de los trabajadores. 
Actualmente, la mayoría de programas de formación en salud laboral, prevención de riesgos laborales y medio ambiente de trabajo están diseñados para ser impartidos de manera presencial y, de hecho, sigue habiendo una cierta resistencia cultural a la formación online en materia de prevención porque se suele considerar de menor calidad que la presencial. Hay que partir de la base de que, en realidad, muchas de estas opiniones están basadas en prejuicios y estereotipos, ya que son numerosas las investigaciones que han puesto de manifiesto la pertinencia de esta metodología de formación. A estas alturas, la enseñanza a distancia ha de considerarse objetivamente, al menos tan eficaz como la presencial (Dean, Stark, Sylwester y Pearl, 2001). Específicamente en el ámbito de la prevención de riesgos laborales, se demuestra en otro trabajo la pertinencia de la formación semipresencial en una experiencia piloto dirigida a delegados de prevención de riesgos laborales, que se evalúa en tres niveles: satisfacción, nivel de conocimientos y transferencia de la formación (Cobos, 2010).

El profesor García Aretio (1989), quien viene defendiendo las posibilidades de esta modalidad formativa desde hace años, opina que la educación a distancia permite democratizar el acceso a la educación, propiciar un aprendizaje autónomo y ligado a la experiencia, impartir una enseñanza innovadora y de calidad, fomentar la educación permanente y reducir los costes. Estos precedentes parecen suficientes para apostar por esta modalidad de formación, teniendo presente también que la enseñanza a distancia plantea numerosos retos en lo que se refiere a la socialización de los participantes y la tutorización de las acciones formativas. Un programa de formación basado en la web ha de ser cuidadosamente concebido y evaluado en términos de objetivos organizativos, de prevención y didácticos. La facilidad de uso es una prioridad (Sorine, Walls y Trinkleback, 2001).

De hecho, muchas de las críticas que se hacen a la formación online realmente no discriminan los programas buenos de los deficientes. Es cierto que hay muchos programas formativos que no están siendo diseñados desde una perspectiva práctica que facilite la interacción y el aprendizaje reflexivo, pero es ahí donde habría que poner el énfasis, sea cual sea la modalidad de impartición de la formación. El foco de la evaluación habría de ponerse en las dos condiciones básicas de significatividad necesarias para que haya un genuino aprendizaje: la coherencia lógica estructural del material didáctico y la adaptación de éste a la estructura cognitiva previa de los participantes (Roselli, 2010).

La formación online no es aplicable a todos los perfiles de participantes ni a todos los sectores ni situaciones aunque, sin duda, se vislumbra un incremento de este tipo de metodologías en los próximos años. Algunas de las tendencias que previsiblemente apuntarán en esta dirección tienen que ver con un incremento del aprendizaje colaborativo basado en herramientas de la web 2.0, un aumento de la formación a distancia sincrónica, gracias a las nuevas herramientas de tele-conferencia, cada vez más sencillas y potentes, o la búsqueda de mejores indicadores que permitan evaluar el retorno de la inversión en formación (Ruiz Mafe y Tronch, 2007). Seguramente el futuro pasa por la adopción de metodologías mixtas o de formación combinada (Paulsson, Ivergard y Hunt, 2005). 
Después de veinte años de utilización de estas tecnologías, puede decirse que la calidad de la formación virtual sigue dependiendo, en gran medida, de su componente pedagógico (García del Dujo y Suárez, 2011). De hecho, en el caso estudiado ha quedado ampliamente demostrado, como se verá más adelante, que la satisfacción con la formación impartida en materia de prevención de riesgos y medio ambiente de trabajo implementado en modalidad online por ISTAS ha sido muy alta con relación al nivel, calidad y funcionamiento de los cursos y con la formación práctica recibida. Además, los participantes reconocen el potencial y utilidad de los nuevos aprendizajes, lo que se refleja en una mentalidad preventiva que antes de la formación no existía.

El objetivo de la investigación es conocer el impacto y satisfacción de los participantes de la formación online impartida por ISTAS durante el año 2008. ISTAS, como entidad asesora, colaboradora e interlocutora de las diferentes organizaciones de Comisiones Obreras (CC.OO.), pone a disposición de estas diversos cursos online y semipresenciales, relacionados con la Salud Laboral y el Medio Ambiente, fundamentalmente en el ámbito del Plan Intersectorial Confederal.

\section{METODOLOGÍA}

\section{Universo de datos y muestra}

Se asume un diseño de investigación cualitativa de estudio de casos múltiple para conocer en detalle las subjetividades de los participantes. Se realizan entrevistas en profundidad a una muestra intencional de los cinco cursos que, a juicio de ISTAS, son los más relevantes de su programación, por lo que interesa una mayor profundización en su estudio. Se entrevistan 184 personas de los 1.300 sujetos que realizan algún curso en el periodo que se estudia. Se distribuyen en cinco grupos correspondientes a los cursos impartidos que se evalúan (ver Tabla 1):

TABLA 1

PARTICIPANTES DE LA INVESTIGACIÓN

\begin{tabular}{|l|c|}
\hline Cursos de formación evaluados & Participantes \\
\hline Prevención de Riesgos Laborales - Nivel Básico & 11 \\
\hline Intervención Sindical & 28 \\
\hline Riesgos Psicosociales en el Entorno Laboral & 40 \\
\hline Sistemas de Gestión Medio Ambiental (SGMA) & 7 \\
\hline Prevención de Riesgos Laborales - Nivel Superior & 98 \\
\hline
\end{tabular}

Las características de los participantes entrevistados se resumen en la Tabla 2: 
TABLA 2

CARACTERÍSTICAS DE LOS PARTICIPANTES ENTREVISTADOS

\begin{tabular}{|c|c|c|c|c|c|c|}
\hline Edad & 26 a 30 años (6\%) & $\begin{array}{c}31 \text { a } 40 \text { años } \\
(44,81 \%)\end{array}$ & $\begin{array}{c}41 \text { a } 50 \text { años } \\
(36,06 \%)\end{array}$ & $\begin{array}{c}51 \text { a } 60 \text { años } \\
(8,74 \%)\end{array}$ & & \\
\hline $\begin{array}{l}\text { Formación } \\
\text { académica }\end{array}$ & $\begin{array}{c}\text { Estudio } \\
\text { Primarios } \\
(1,12 \%)\end{array}$ & $\begin{array}{c}\text { Estudios } \\
\text { Secundarios } \\
(1,12 \%)\end{array}$ & $\begin{array}{l}\text { Bachillerato/ } \\
\operatorname{COU}(7,82 \%)\end{array}$ & $\begin{array}{l}\text { F.P. Grado } \\
\text { Medio }(4,47 \%)\end{array}$ & $\begin{array}{c}\text { F.P. Grado } \\
\text { Superior } \\
(7,26 \%)\end{array}$ & $\begin{array}{c}\text { Enseñanzas } \\
\text { Superiores } \\
(78,21 \%)\end{array}$ \\
\hline $\begin{array}{l}\text { Perfil } \\
\text { sindical }\end{array}$ & $\begin{array}{l}\text { Delegado o } \\
\text { Cuadro Sindical } \\
(40,79 \%)\end{array}$ & $\begin{array}{l}\text { Afiliado } \\
(28,36 \%)\end{array}$ & $\begin{array}{l}\text { Sin relación con } \\
\text { CC.0O. }(20,89 \%)\end{array}$ & $\begin{array}{c}\text { Otros perfiles } \\
\text { sindical }(9,95 \%)\end{array}$ & & \\
\hline $\begin{array}{c}\text { Sector de } \\
\text { trabajo }\end{array}$ & $\begin{array}{l}\text { Administración } \\
\text { Pública }(45,51 \%)\end{array}$ & $\begin{array}{l}\text { Sanidad } \\
(16,29 \%)\end{array}$ & $\begin{array}{c}\text { Enseñanza } \\
(15,15 \%)\end{array}$ & Otros $(23,05)$ & & \\
\hline $\begin{array}{l}\text { Antigüedad } \\
\text { en el sector }\end{array}$ & $\begin{array}{c}0 \text { a } 5 \text { años } \\
(22,40 \%)\end{array}$ & $\begin{array}{c}\text { 6 a } 10 \text { años } \\
(21,86 \%)\end{array}$ & $\begin{array}{c}11 \text { a } 15 \text { años } \\
(13,66 \%)\end{array}$ & $\begin{array}{c}16 \text { a } 20 \text { años } \\
(23,59 \%)\end{array}$ & $\begin{array}{c}21 \text { años en } \\
\text { adelante } \\
(18,58 \%)\end{array}$ & \\
\hline $\begin{array}{c}\text { Cargo } \\
\text { desarrollado }\end{array}$ & $\begin{array}{l}\text { Ejecutivo } \\
(2,73 \%)\end{array}$ & $\begin{array}{c}\text { Cargo } \\
\text { intermedio } \\
(18,03 \%)\end{array}$ & $\begin{array}{c}\text { Operarios } \\
(44,81 \%)\end{array}$ & $\begin{array}{c}\text { Auxiliares } \\
\text { administrativos } \\
(27,97 \%)\end{array}$ & $\begin{array}{c}\text { Desempleados } \\
(6 \%)\end{array}$ & \\
\hline $\begin{array}{l}\text { Tipología de } \\
\text { empresa }\end{array}$ & $\begin{array}{c}\text { Gran empresa } \\
\qquad(68,45 \%)\end{array}$ & $\begin{array}{c}\text { Medianas } \\
\text { empresas } \\
(15,48 \%)\end{array}$ & $\begin{array}{c}\text { Pequeñas } \\
\text { empresas }(9,52 \%)\end{array}$ & $\begin{array}{c}\text { Microempresas } \\
(6,55 \%)\end{array}$ & & \\
\hline $\begin{array}{l}\text { Cursos } \\
\text { realizados }\end{array}$ & $\begin{array}{c}\text { Un único curso } \\
(62 \%)\end{array}$ & $\begin{array}{l}\text { Más de un } \\
\text { curso }(37,16 \%)\end{array}$ & & & & \\
\hline
\end{tabular}

\section{Instrumento de recogida de datos}

El instrumento para la recogida de datos es la entrevista estructurada y en profundidad. Esta se administra sobre los 184 sujetos seleccionados utilizando el chat escrito de la plataforma tecnológica de ISTAS, creada por la empresa XIP Multimèdia. El diseño de la entrevista trata de dar respuesta al objetivo principal que es conocer el grado de satisfacción e impacto de la formación recibida sobre la actividad profesional y laboral desempeñada.

\section{Tratamiento de los datos}

Una vez se administra las entrevistas, los datos se organizan atendiendo a los cinco cursos estudiados y se preparan para su posterior análisis con el programa AQUAD 6.0. El Gráfico 1 resume el procedimiento realizado: 


\begin{tabular}{|c|c|c|c|}
\hline RECOGIDA & $\triangle$ ORGANIZACION & PREPARACION & PROYECTO \\
\hline 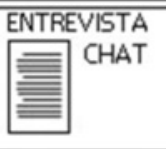 & $\begin{array}{l}\text { CURSOS } \\
\text { 1. Básico } \\
\text { 2. Interv. Sind. } \\
\text { 3. Psicosociales } \\
\text { 4. SGMA } \\
\text { 5. Superior }\end{array}$ & $\begin{array}{l}\text { NOMENCLATURA } \\
\text { MAQUETACIONN } \\
\text { FORMATO (RTF) }\end{array}$ & $\begin{array}{l}\text { AQUAD } \\
\text { Proyecto ISTAS } \\
\text { 1. Catal. archivos } \\
\text { 2. Catal. codigos } \\
\text { CODIFICACION }\end{array}$ \\
\hline
\end{tabular}

GRÁFICO 1

TRATAMIENTO DE DATOS EN LA FASE CUALITATIVA DE ENTREVISTAS

\section{Proceso de codificación}

La tarea de codificación se desarrolla en distintas fases, de manera progresiva e individual sobre las mismas remesas de entrevistas. Se toman decisiones que modelan el esquema de códigos a utilizar y la configuración de la estructura de las distintas versiones del sistema de categorías. Para el ejercicio de la codificación, se toma como unidad de análisis la respuesta completa del entrevistado, pudiendo realizar una codificación múltiple, asociando diversos códigos a la respuesta. Como resultado de esta primera fase en el análisis, esta forma de codificación produce más de 5.500 códigos.

Finalizada la fase de codificación y establecido el método de análisis de contenido para lograr los resultados deseados, se suceden procesos continuos de búsquedas específicas, recuperación selectiva de datos y su organización por categorías para dar paso a la subsiguiente tarea de interpretación. Este tipo de análisis supuso trabajar sobre una ingente cantidad de datos organizados al objeto de extraer y presentar aquellas ideas relevantes para el estudio, que fueron expresadas con una frecuencia significativa. En todo momento se tuvo especial cautela y sensibilidad en la interpretación de los datos para no generar ideas sesgadas.

\section{Proceso de creación del sistema de categorías y del cálculo de la fiabilidad}

En el proceso de construcción del sistema de categorías para evaluar la formación online de ISTAS intervienen tres codificadores. Se utiliza el programa Fleiss v.1.0 con el objetivo de conocer el valor de la concordancia (Fleiss, 1981). El resumen del proceso del cálculo del coeficiente Kappa de Fleiss $(\mathrm{K})$ se realiza en dos momentos, como se observa en el Gráfico 2. Los dos coeficientes que se obtienen son $K=0,852$ y $K=0,893$. La fiabilidad de los resultados obtenidos se apoya en un trabajo previo sobre concordancia entre codificadores empleado por Torres y Perera (2009).

El sistema de categorías empleado se crea a partir de cuatro borradores previos. La primera versión se genera de forma deductiva, considerando las preguntas de las entrevistas y de los objetivos de la evaluación. Las versiones posteriores siguen un proceso inductivo, tomando los datos de las entrevistas. El sistema de codificación definitivo se compone de 4 dimensiones, 18 categorías, 9 subcategorías y 135 indicadores (ver Tabla 3). 


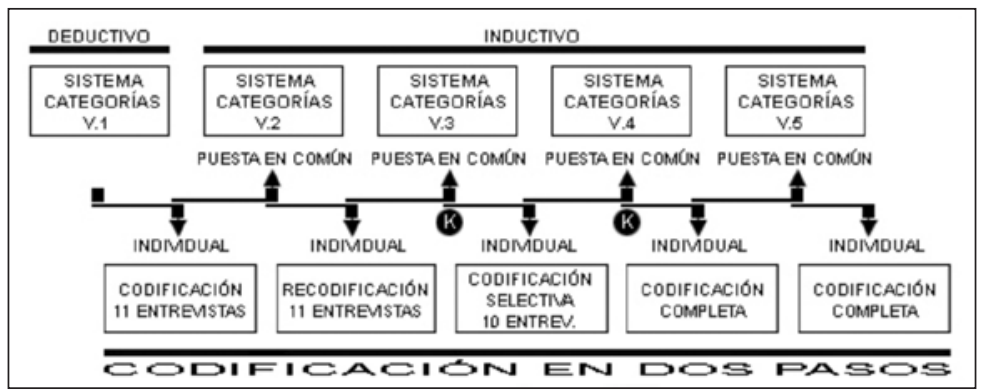

GRÁFICO 2

HISTÓRICO DE CONSTRUCCIÓN DEL SISTEMA DE CATEGORÍAS DEFINITIVO

TABLA 3

SISTEMA DE CATEGORÍAS DEFINITIVO DE LA INVESTIGACIÓN PRESENTADA

\begin{tabular}{|c|c|c|}
\hline \multicolumn{3}{|c|}{ SISTEMA DE CATEGORÍAS PARA EVALUAR LA FORMACIÓN ONLINE DE ISTAS } \\
\hline CATEGORÍAS & INDICADORES & CÓDIGOS \\
\hline \multicolumn{3}{|c|}{ DIMENSIÓN: RASGOS SOCIODEMOGRÁFICOS } \\
\hline \multirow{6}{*}{ Edad } & $18-25$ & E1 \\
\hline & $26-30$ & E2 \\
\hline & $31-40$ & E3 \\
\hline & $41-50$ & E4 \\
\hline & $51-60$ & E5 \\
\hline & Más de 60 & E6 \\
\hline \multirow{19}{*}{$\begin{array}{l}\text { Comunidad } \\
\text { Autónoma }\end{array}$} & Andalucía & AAND \\
\hline & Aragón & AARA \\
\hline & Asturias & AAST \\
\hline & Canarias & AICA \\
\hline & Cantabria & ACAN \\
\hline & Castilla y León & ACAL \\
\hline & Castilla-La Mancha & ACAM \\
\hline & Cataluña & ACAT \\
\hline & Ceuta & ACEU \\
\hline & Extremadura & AEXT \\
\hline & Galicia & AGAL \\
\hline & Islas Baleares & AISB \\
\hline & La Rioja & ALRI \\
\hline & Madrid & AMAD \\
\hline & Melilla & AMEL \\
\hline & Murcia & AMRU \\
\hline & Navarra & ANAV \\
\hline & País Vasco & APAV \\
\hline & Valencia & AVAL \\
\hline
\end{tabular}




\begin{tabular}{|c|c|c|}
\hline \multicolumn{3}{|c|}{ SISTEMA DE CATEGORÍAS PARA EVALUAR LA FORMACIÓN ONLINE DE ISTAS } \\
\hline CATEGORÍAS & INDICADORES & CÓDIGOS \\
\hline \multicolumn{3}{|c|}{ DIMENSIÓN: RASGOS SOCIODEMOGRÁFICOS } \\
\hline \multirow{6}{*}{$\begin{array}{l}\text { Formación } \\
\text { Académica }\end{array}$} & Estudios Primarios & FPRI \\
\hline & Estudios Secundarios & FSEC \\
\hline & Bachillerato / COU & FBAC \\
\hline & F.P. Grado Medio & FPGM \\
\hline & F.P. Grado Superior & FPGS \\
\hline & $\begin{array}{l}\text { Enseñanzas Universitarias (Ldo.; Máster y } \\
\text { Doctorado) }\end{array}$ & FUNI \\
\hline \multirow{5}{*}{ Perfil sindical } & Delegado Sindical o Miembro Comité Empresa & PDEL \\
\hline & Cuadro Sindical & PCUS \\
\hline & Afiliado & PAFI \\
\hline & Sin relación con CC.OO. & PSIN \\
\hline & Otros & POTR \\
\hline \multirow{10}{*}{ Sector de trabajo } & Agroalimentaria & SAGR \\
\hline & Administración Pública & SADM \\
\hline & Servicios financieros y administrativos & SSER \\
\hline & Construcción y Madera & SCON \\
\hline & Textil y Químicas & STEX \\
\hline & Enseñanza & SENS \\
\hline & Comercio, Hotelería y Turismo & SHOT \\
\hline & Minero Metalúrgica & SMIN \\
\hline & Sanidad & SSAN \\
\hline & Comunicación y Transporte & STRA \\
\hline \multirow{5}{*}{$\begin{array}{l}\text { Antigüedad en el } \\
\text { sector }\end{array}$} & $0-5$ & A1 \\
\hline & $6-10$ & $\mathrm{~A} 2$ \\
\hline & $11-15$ & A3 \\
\hline & $16-20$ & A4 \\
\hline & 21- en adelante & A5 \\
\hline \multirow{5}{*}{ Cargo desarrollado } & Auxiliar de servicios & CAUX \\
\hline & Operativo & COPE \\
\hline & Mando intermedio & CMAN \\
\hline & Ejecutivo & CEJE \\
\hline & Desempleado & CPAR \\
\hline \multirow{4}{*}{$\begin{array}{l}\text { Tipología de } \\
\text { empresa }\end{array}$} & Microempresa & TMIC \\
\hline & Pequeña empresa & TPEQ \\
\hline & Mediana empresa & TMED \\
\hline & Gran empresa & TGRA \\
\hline \multirow{2}{*}{ Cursos hechos } & Uno & CUNO \\
\hline & Más de uno & CMAS \\
\hline
\end{tabular}




\begin{tabular}{|c|c|c|c|c|}
\hline \multicolumn{5}{|c|}{ SISTEMA DE CATEGORÍAS PARA EVALUAR LA FORMACIÓN ONLINE DE ISTAS } \\
\hline CATEGORÍAS & SUBCATEGORÍAS & INDICADORES & \multicolumn{2}{|c|}{ CÓDIGOS } \\
\hline \multicolumn{5}{|c|}{ CATEGORÍA: IMPACTO } \\
\hline \multirow{16}{*}{$\begin{array}{l}\text { Elementos } \\
\text { del curso }\end{array}$} & \multirow{8}{*}{$\begin{array}{c}\text { General: } \\
\text { No cambiaría, gusta } \\
\text { mucho }\end{array}$} & Objetivos & \multirow{8}{*}{$\begin{array}{l}\sum_{11} \\
0 \\
0 \\
\text { II }\end{array}$} & IEOBJ1 \\
\hline & & Contenidos & & IECON1 \\
\hline & & Metodología & & IEMET1 \\
\hline & & Materiales & & IEMAT1 \\
\hline & & Actividades & & IEACT1 \\
\hline & & Tutorización & & IETUT1 \\
\hline & & Temporización & & IETEM1 \\
\hline & & Evaluación & & IEEVA1 \\
\hline & \multirow{8}{*}{$\begin{array}{c}\text { General: } \\
\text { Modificaciones, } \\
\text { cambiaría }\end{array}$} & Objetivos & \multirow{8}{*}{ 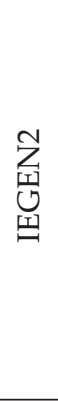 } & IEOBJ2 \\
\hline & & Contenidos & & IECON2 \\
\hline & & Metodología & & IEMET2 \\
\hline & & Materiales & & IEMAT2 \\
\hline & & Actividades & & IEACT2 \\
\hline & & Tutorización & & IETUT2 \\
\hline & & Temporización & & IETEM2 \\
\hline & & Evaluación & & IEEVA2 \\
\hline \multirow{10}{*}{ Real } & \multirow{7}{*}{ Aplicación directa } & Actuación directa & & IRADI \\
\hline & & Consciencia & & IRCON \\
\hline & & Ayuda a otros & & IRAYU \\
\hline & & Seguir estudiando & & IREST \\
\hline & & Inconsciencia & & IRINC \\
\hline & & Seguridad laboral & & IRSEG \\
\hline & & Ninguna & & IRNIN \\
\hline & \multirow{3}{*}{$\begin{array}{l}\text { Necesidades } \\
\text { cubiertas }\end{array}$} & Cambio actitud & & IRCAM \\
\hline & & Relación con otros & & IRREL \\
\hline & & Conocimientos & & IRCTO \\
\hline \multirow{8}{*}{\multicolumn{2}{|c|}{ Aspecto más positivo }} & Cambio actitud & & IPCAM \\
\hline & & Titulación & & IPTIT \\
\hline & & Seguridad laboral & & IPSEG \\
\hline & & Contacto tutores & & IPTUT \\
\hline & & Modalidad formativa & & IPMOD \\
\hline & & Comunicación & & IPCOM \\
\hline & & Metodología & & IPMET \\
\hline & & Nuevos contenidos & & IPCON \\
\hline
\end{tabular}




\begin{tabular}{|c|c|c|c|c|}
\hline \multicolumn{5}{|c|}{ SISTEMA DE CATEGORÍAS PARA EVALUAR LA FORMACIÓN ONLINE DE ISTAS } \\
\hline CATEGORÍAS & SUBCATEGORÍAS & INDICADORES & \multicolumn{2}{|c|}{ CÓDIGOS } \\
\hline \multicolumn{5}{|c|}{ CATEGORÍA: IMPACTO } \\
\hline \multirow{11}{*}{$\begin{array}{l}\text { Expectativas no } \\
\text { cumplidas }\end{array}$} & \multirow{7}{*}{ No aporta el curso } & Casos prácticos & & INCAS \\
\hline & & Normativa & & INNOR \\
\hline & & Evaluación riesgos & & INFAC \\
\hline & & Herramientas & & INHER \\
\hline & & Especialización & & INEST \\
\hline & & Prácticas reales & & INPRA \\
\hline & & Ninguna & & INNIN \\
\hline & $\begin{array}{l}\text { Posibilidad de } \\
\text { trabajarlo }\end{array}$ & $\begin{array}{l}\text { Readaptación de } \\
\text { elementos curso }\end{array}$ & & INREA \\
\hline & \multirow{3}{*}{$\begin{array}{l}\text { Propuestas y cómo } \\
\text { trabajarlo }\end{array}$} & Más tiempo & & INTEM \\
\hline & & Más contenidos & & INCON \\
\hline & & Metodología & & INMET \\
\hline \multirow{7}{*}{\multicolumn{2}{|c|}{ Dificultades }} & Tiempo & & IDTIE \\
\hline & & Organización & & IDORG \\
\hline & & Aplicación empresa & & IDAPL \\
\hline & & Adaptación & & IDADA \\
\hline & & Resistencia & & IDRES \\
\hline & & Otras & & IDOTR \\
\hline & & Ninguna & & IDNIN \\
\hline \multirow{3}{*}{\multicolumn{2}{|c|}{ Nivel Satisfacción }} & Complacido & & SCOM \\
\hline & & Satisfecho & & SSAT \\
\hline & & Insatisfecho & & SINS \\
\hline \multirow{16}{*}{$\begin{array}{l}\text { Elementos del } \\
\text { curso }\end{array}$} & \multirow{8}{*}{$\begin{array}{l}\text { General: } \\
\text { Satisfactorio }\end{array}$} & Objetivos & \multirow{8}{*}{$\begin{array}{l}\vec{Z} \\
\overrightarrow{\mathrm{T}} \\
\mathrm{D} \\
\text { 蒠 }\end{array}$} & SEOBJ1 \\
\hline & & Contenidos & & SECON1 \\
\hline & & Metodología & & SEMET1 \\
\hline & & Materiales & & SEMAT1 \\
\hline & & Actividades & & SEACT1 \\
\hline & & Tutorización & & SETUT1 \\
\hline & & Temporización & & SETEM1 \\
\hline & & Evaluación & & SEEVA1 \\
\hline & \multirow{8}{*}{$\begin{array}{l}\text { General: } \\
\text { No satisfactorio }\end{array}$} & Objetivos & \multirow{8}{*}{ 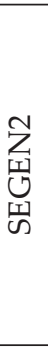 } & SEOBJ2 \\
\hline & & Contenidos & & SECON2 \\
\hline & & Metodología & & SEMET2 \\
\hline & & Materiales & & SEMAT2 \\
\hline & & Actividades & & SEACT2 \\
\hline & & Tutorización & & SETUT2 \\
\hline & & Temporización & & SETEM2 \\
\hline & & Evaluación & & SEEVA2 \\
\hline \multicolumn{2}{|c|}{ Agradecimientos } & Agradecimientos & & OAGRA \\
\hline \multicolumn{2}{|c|}{ Ampliar oferta formativa } & Ampliar oferta formativa & & OAFOR \\
\hline
\end{tabular}




\section{RESULTADOS}

En este apartado se ofrecen los resultados más destacados presentados por categorías (ver Gráfico 3). En primer lugar se atiende a la influencia y las mejoras del diseño de los elementos de la programación sobre los distintos cursos de formación evaluados. Después se muestran las necesidades cubiertas en el impacto de la formación, las expectativas no cumplidas y la satisfacción de los participantes con la formación. Finalmente se presentan el impacto real sobre el desempeño laboral, los aspectos relevantes del impacto de la formación y las dificultades en el impacto.

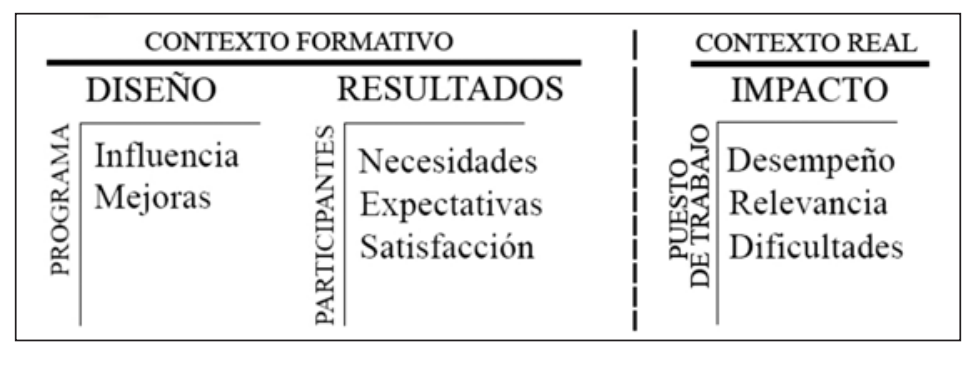

\section{GRÁFICO 3 \\ ÁMBITOS DE EVALUACIÓN}

\section{Influencia y mejoras del diseño de la formación}

Los objetivos (IEOBJ) se definen como claros, flexibles y adecuados. El planteamiento de los fines que seguía la formación teórica se cubre gracias a la orientación práctica del curso. Los contenidos (IECON) resultan claros, comprensibles, completos y fáciles de asimilar, de modo que ofrecen una perspectiva amplia del tema de estudio. La metodología (IEMET) que se emplea genera un correcto desarrollo en los procesos de aprendizaje. Los aspectos que mejor la definen son: práctica, dinámica, participativa, flexible y adecuada. Los materiales (IEMAT), manuales y revistas se evalúan positivamente en su vertiente didáctica y utilitaria. Las actividades (IEACT) resultan clarificadoras y apropiadas, a veces breves y concisas, centradas en casos prácticos.

"En general el curso me ha parecido de mucha calidad en todos sus elementos, añadido a la flexibilidad en la profundización en cada tema que cada uno quisiese". (Entrevista sgma.004)

La evaluación (IEEVA) y autoevaluación se perciben como una actividad práctica y de seguimiento que permiten valorar y afianzar los aprendizajes. La temporización (IETEM) es práctica y adecuada durante el desarrollo del curso y adaptada a la situación laboral de los participantes. La tutorización (IETUT) es satisfactoria, destacando la rápida atención individualizada y la resolución de las dudas y consultas formuladas; el planteamiento continuo de reflexiones; el seguimiento, 
apoyo y orientación que se presta durante el proceso de aprendizaje; el trato de motivación y confianza que se da al participante; y la disponibilidad permanente para comunicarse con el tutor.

"El método de evaluación también me pareció válido sobre todo el que se hicieran ejercicios más prácticos que teóricos". (Entrevista super.031)

En cuanto a las mejoras que se proponen en la programación de la formación, un primer aspecto es la readaptación de los elementos del curso (INREA), de modo que alcance un mayor impacto. Hay una necesidad de mejorar la planificación de los tiempos (INTEM), por la complejidad de compaginarlo con otros deberes. Se propone diseñar nuevos casos prácticos (INCON) sobre supuestos reales y relacionar toda la documentación en torno a cómo resolverlos. Se sugiere trabajar en grupo (INMET) sobre casos prácticos para que se puedan conocer las ideas, opiniones y experiencias de todos los participantes; y, se propone que se utilicen más medios audiovisuales y esquemas que mejoren la comprensión de los temas.

\section{"[...] enfocaría los cursos de manera más especializada". (Entrevista insin.005)}

\section{Necesidades, expectativas y satisfacción de la formación}

Hay un cambio de actitud (IRCAM) en el trabajo que los participantes desarrollan. Los participantes comentan su interés por promover un tipo de relación laboral más cercana y directa con sus propios compañeros (IRREL). La actualización y ampliación de los conocimientos prácticos (IRCTO) les capacita para abordar la realidad de las condiciones en el trabajo.

"Sobre todo, el curso me ha hecho ser más consciente de lo importante que es la Seguridad en el Trabajo y a saber detectar ciertos Riesgos que me rodean en mí día a día". (Entrevista super.082)

El curso es satisfactorio, cubriendo las expectativas de todos los participantes (INNIN). No obstante, se reconocen determinadas situaciones de riesgo, aunque no se sabe combatirlos en la práctica (INCAS) por falta de nuevos casos prácticos. No se trabaja adecuadamente el aprendizaje sobre normativas (INNOR). Se requiere más dedicación a la evaluación y solución de los factores de riesgos psicosociales (INFAC). Son necesarias nuevas estrategias y recursos que avalen la seriedad y eficacia en el trabajo de la prevención de riesgos laborales (INHER). Algunos contenidos no tienen relación con el sector laboral de los participantes (INEST). Los cursos fueron áridos en contenidos $y$, en consecuencia, se requiere más formación en prácticas reales y aplicaciones directas en la empresa (INPRA).

"La ampliación de casos prácticos estaría muy bien. [...] Trabajar casos particulares de los participantes sería mejor, pero, por otro lado, muy complicado para los docentes". (Entrevista psico.024) 
Hay una percepción unánime que expresa, en general, una alta satisfacción (SCOM) hacia el nivel, la calidad y el funcionamiento del curso y, en particular, hacia la formación práctica recibida. Los aspectos positivos destacados son (SEGEN1): la organización; los contenidos, material y otros recursos; la formación práctica y aplicabilidad; la comunicación con los compañeros y la tutorización y metodología. Los aspectos negativos son (SEGEN2): mejorar los tests; algunas actuaciones concretas de los tutores (dinamizar el chat, rapidez en las respuestas, tutorización personalizada, etc.); la programación y temporización.

"Me ha satisfecho mucho. He aprendido cosas importantes que se pueden dar en mi puesto de trabajo y antes no les daba importancia. Todo lo que esperaba del curso, lo he encontrado en él". (Entrevista basic.003)

Impacto, relevancia y dificultades de la formación sobre el desempeño laboral

Los participantes comentan no tener oportunidad para aplicar lo aprendido por motivo de excedencia o porque actualmente no desarrollan ninguna actividad preventiva en su centro de trabajo (IRNIN). Sin embargo, la formación recibida por los participantes influye en la manera cómo perciben y actúan en materia de PRL $\mathrm{y}$, en consecuencia, esto produce un impacto directo e inmediato en su entorno de trabajo. En general, los participantes aplican completamente lo aprendido (al nivel de lo que su responsabilidad le permite), otras veces la aplicación es parcial (cuando solo es necesario, debido a la adecuación de los puestos de trabajo, por limitaciones presupuestarias), y en escasas ocasiones no tienen opción a aplicarlo directamente (IRADI). Los participantes buscan la implicación y cooperación con sus compañeros (sugiriendo procedimientos y aportando soluciones a algunos problemas) para atender sus necesidades. La cooperación consiste en establecer un trato directo y mantener conversaciones entre todos antes de tomar decisiones que afectan a la seguridad y salud de los trabajadores (IRAYU).

" [...] El impacto no está relacionado con mi trabajo sino con mi formación que se ha visto muy beneficiada con la realización del curso". (Entrevista super.048)

Los participantes manifiestan obtener una mayor información, referentes, guías (normativa), conocimientos, reflexión, comprensión y nueva perspectiva (visión preventiva), enfoque más técnico de cómo aplicar lo aprendido y nuevos argumentos legislativos (IRCON). La mayoría coincide que el valor de la formación se refleja en la mejora que supone el impacto real del trabajo que realiza (IREST). Son pocos participantes los que, recibiendo la formación, comentan no poner en práctica lo aprendido (IRINC). Los participantes aseguran tener un mayor conocimiento de la normativa y mantienen una actitud de observación y actuación sobre los posibles riesgos (IRSEG).

"En este caso creo que como se puede ver el impacto real en mi caso es bastante alto, y serían bastante los ejemplos que he podido poner en práctica [...]". (Entrevista psico.004) 
Manifiestan asimismo alcanzar un mayor conocimiento sobre legislación en PRL, así también sobre la normativa vigente, y en técnicas y herramientas que les sirven para detectar deficiencias en sus puestos de trabajo a fin de realizar propuestas de mejora con una mayor seguridad (IPCON). Los participantes comentan tener un mayor concienciación personal y social en la importancia de la prevención (mentalidad preventiva) (IPCAM). La realización de los cursos de formación en PRL proporciona los conocimientos necesarios (e incluso su ampliación) para poder desempeñar una mejor actuación en el ámbito laboral, consolidándoles en el mismo o posibilitándoles el acceso a mejoras laborales, ya que son un requisito (IPTIT). Valoran positivamente el método de enseñanza dinámica basada en las herramientas de comunicación (chat y foros) para fomentar la interacción y el trabajo en grupo (IPCOM). Los cursos se adaptan adecuadamente a las necesidades de los usuarios, y no a la inversa. Valoran positivamente las exposiciones claras y didácticas de los materiales proporcionados, así como las prácticas y actividades realizadas (IPMET). El método de enseñanza les permite acceder a la formación para compaginar y conciliar su vida laboral y personal (IPMOD). Manifiestan un cambio en el trabajo a raíz de la adquisición de los conocimientos, estrategias y herramientas en el campo de la PRL (IPSEG). El alto grado de satisfacción con los tutores, pone de relieve la amabilidad, rapidez y dominio de la materia. Destacan la capacidad pedagógica de estos para generar oportunidades que ayudan a reflexionar y fomentar el aprendizaje individual (IPTUT).

"Me ha dado una visión más amplia acerca de cómo afrontar determinados problemas relacionados no solo con el puesto de trabajo, sino también con los propios compañeros". (Entrevista insin.025)

La mayoría cree poder llevarlo a la práctica sin dificultad. No obstante, las dificultades dependen más del marco interno de relaciones laborales en el centro que de las propias acciones que plantea el propio curso (IDNIN). Sin embargo, hay una coincidencia en la opinión de querer flexibilizar la programación y los plazos de entrega de las actividades de modo que sean compatibles con la situación personal y laboral Tiempo (IDTIE). Lo más relevante: el curso es demasiado extenso; hubo problemas con la empresa que repartió el material y la no adecuación de la formación a la especificidad del sector de trabajo (IDORG). Se detectan tres tipos de problemas que dificultan que puedan llegar a aplicar lo aprendido en la empresa: el trato con los superiores, el coste económico que supone y la propia estructura y funcionamiento de la Administración (IDAPL). La necesidad de que los contenidos estén adaptados a niveles más específicos de cada sector laboral para que los aprendizajes se puedan llevar a la práctica (IDADA). La primera barrera son los propios trabajadores. La PRL es una cuestión que creen difícil de comprender por sus compañeros, y más aún por aquellos que llevan más tiempo trabajando (los mayores). Los hábitos están muy marcados e intentan modificar estas conductas en un contexto complicado de desmotivación y burocratización. Por otra parte, suele existir una estructura jerárquica muy fuerte (trabajador-jefe) y los cambios son fáciles en el nivel de tu propia responsabilidad. Es necesario trabajar estrategias que ayuden a mejorar la comunicación y las relaciones personales (IDRES). En orden de frecuencia, algunas de las dificultades detectadas son: 
el desplazamiento para realizar los exámenes presenciales en otras sedes/delegaciones distintas a Madrid; el hecho de estar en el paro hace imposible aplicar a la práctica lo que se aprende; y un mayor apoyo de la organización del sindicato en PRL en materia de información (IDOTR).

"Quizás lo único que cambiaría sería flexibilizar un poco más la temporización de las evaluaciones para adaptarlas mejor a las disponibilidades horarias de cada trabajador". (Entrevista basic.009)

\section{CONCLUSIONESY PROPUESTAS DE MEJORA}

Uno de los logros destacados de la formación recibida tiene que ver con el cambio de las actitudes con respecto a la práctica laboral. En la medida de sus posibilidades y competencias, los participantes ahora que están formados actúan con mentalidad preventiva en sus empresas. En el desarrollo de la práctica profesional, los contenidos adquieren un sentido utilitario que permite ampliar y actualizar los conocimientos sobre una materia concreta, identificar los problemas y saber cómo solucionarlos. Hay que tener en cuenta que mientras mayor participación e implicación se pide a los trabajadores en la formación, mayor impacto se produce (Wallen y Mulloy, 2006a). Además, en investigaciones evaluativas de programas de formación se comprueba, tal como ocurre en nuestro estudio, que otro aspecto que contribuye a favorecer el impacto de la formación recibida tiene que ver con la valoración que los participantes hacen sobre el diseño de las acciones formativas (Buendía, Expósito y Sánchez, 2012).

Las metodologías que involucran al trabajador mediante una cantidad importante de actividad práctica son las más efectivas para la formación en materia de prevención de riesgos laborales (Burke et al., 2006). En este sentido, la metodología desarrollada por ISTAS se caracteriza como favorable para organizar y controlar fácilmente el propio tiempo, facilitando la compatibilización de los estudios con otras responsabilidades familiares y/o profesionales. El valor de las actividades desarrolladas radica en que son consideradas ejemplos de prácticas reales que pueden trasladarse al terreno profesional. Siguiendo esta línea, si hubiera que hacer una propuesta de mejora en este ámbito sería indudablemente la de profundizar aún más en la dotación a los trabajadores del conocimiento y la capacidad para rediseñar sus propios puestos como herramienta para mejorar el entorno y su percepción de la salud (Robertson, Huang, O’Neill y Schleifer, 2008).

La satisfacción de los participantes no se ha visto afectada por el hecho de que sean conscientes de la dificultad que supone aplicar lo aprendido en su ámbito de trabajo y, además, distinguen la formación que reciben del curso respecto a la posible aplicación de lo aprendido. No obstante, esta satisfacción sí está condicionada al deseo de los participantes de expresar las posibles mejoras que podrían incrementar el impacto de la formación recibida en el ámbito laboral. Esta toma de conciencia implica actuar con una visión más objetiva, y con mayor seguridad y capacidad ante los riesgos laborales. Manifiestan un cambio en las pautas de comportamiento que les lleva a estar más abiertos a valorar el trabajo de los demás. En cualquier caso, se concluye con la voluntad de aplicar los nuevos conocimientos y destrezas adquiridas en un futuro o de darle 
alguna aplicación práctica a lo aprendido. Todo ello ha derivado en la posibilidad de exigir responsabilidades a la dirección competente y lograr una mayor sensibilización en los compañeros.

Los participantes piden enfocar mucho más los contenidos al trabajo diario y, con ello, ampliar los supuestos reales/casos prácticos y conocer cómo analizarlos y resolverlos. Esta petición es congruente con las buenas prácticas descritas en la bibliografía especializada. La formación tiene que descender a las características concretas de cada puesto de trabajo. Puede haber módulos generales de formación básica pero además los contenidos han de adaptarse necesariamente a los distintos tipos de trabajo desarrollados (Bena, Berchialla, Coffano, Debernardi y Icardi, 2009). La recomendación sería pues, adaptar mucho más los contenidos de la formación a la práctica profesional de cada puesto de trabajo. El diseño de los contenidos debería enfocarse hacia el ámbito empresarial o administrativo de los participantes, atendiendo a la especificidad contextual del sector y actividad profesional que corresponda en cada caso. En este sentido, la importancia del contexto es apuntada por Buendía, Olmedo y González (2009) quienes lo consideran como "algo inseparable de las acciones humanas y, por tanto, el funcionamiento mental y el marco sociocultural son entendidos como momentos dialécticos que interactúan en toda la acción humana" (p.187).

Como se ha visto a lo largo del artículo, la formación ha cumplido altamente con las expectativas de los participantes, aunque lógicamente siempre es posible hacer algunas recomendaciones que incidan en su mejora. Aparte de las que ya hemos señalado, es posible incidir en dos aspectos más: la duración de los programas formativos y el enriquecimiento audiovisual y gráfico de los materiales didácticos utilizados. En cuanto al primero de ellos, hay que insistir en que no hay evidencias de que los programas formativos de larga duración sean más efectivos que los de corta duración. Parece recomendable que, atendiendo a las características de los participantes, la formación sea de corta duración. Es más interesante una formación inicial sencilla que luego se va reiterando, complementando y mejorando con el tiempo que una intensa formación inicial que luego ya no se repite, recuerda o amplía (Johnson, 2008).

Respecto al tema de los materiales didácticos, la forma en que se presenta la formación lógicamente incide en los resultados de aprendizaje, mejorando notablemente cuando se incluyen imágenes, presentaciones o vídeos (Gagnon, 2005; Gotsch, 1994; Wallen y Mulloy, 2006b; Weidner, Delnevo, Gotsch, y Rosen, 2001). Se ha demostrado empíricamente la teoría de la codificación e información dual que, a efectos didácticos, se concreta en que la información presentada de manera concurrente presenta más oportunidades de construir conexiones en la memoria de largo plazo (Wallen y Mulloy, 2006b).

Todas estas sugerencias prácticas que apuntamos, nacidas de la investigación y la evidencia científica, deben ser un acicate que contribuya a mejorar los materiales y organización de los cursos ya que, como hemos visto anteriormente, conectan muy directamente con las aportaciones y sugerencias de los propios participantes. Esperamos que dichas recomendaciones ayuden a contribuir al proceso de mejoramiento continuo de una formación de impacto tanto en la Fundación ISTAS como en otras entidades que trabajan en este mismo ámbito, en un tema tan sensible y estratégico como es la seguridad y salud de los trabajadores en el ámbito laboral. 


\section{AGRADECIMIENTOS}

A la Fundación ISTAS por su compromiso con el proyecto y especialmente a Vicente López, Valeria Uberti, Lola García y Virginia Saz por su expreso y constante apoyo. Este trabajo forma parte del Contrato de I+D "Programas de formación en prevención de riesgos laborales: Evaluación de impacto y propuestas de Educación para la Salud en el medio laboral", suscrito entre la Universidad Pablo de Olavide y la Fundación Instituto Sindical de Trabajo, Ambiente y Salud (ISTAS), al amparo del artículo 83 de la LOU.

\section{BIBLIOGRAFÍA}

Bena, A., Berchialla, P., Coffano, M. E., Debernardi, M. L. \& Icardi, L. G. (2009). Effectiveness of the training program for workers at construction sites of the highspeed railway line between Torino and Novara: impact on injury rates. American Journal of Industrial Medicine, 52(12), 965-972.

Buendía, L., Olmedo, E. M., \& González, G. (2009). Lifelong Learning: Diferentes contextos, diferentes situaciones. Revista de Investigación Educativa, 27(1), 185-202.

Buendía, L., Expósito, J., \& Sánchez, M. (2012). Investigación evaluativa de programas de formación profesional para el empleo en el ámbito local. Revista de Investigación Educativa, 30(1), 161-179.

Burke, M. J., Sarpy, S. A., Smith-Crowe, K., Chan-Serafin, S., Salvador, R. O., \& Islam, G. (2006). Relative effectiveness of worker safety and health training methods. American Journal of Public Health, 96(2), 315-324.

Carretero, M. A. (2006). Metodología, procedimientos, estrategias y actividades de formación continua y ocupacional en modalidad e-Learning. En A. Alvarado \& A. Rodríguez (Eds.), La formación sin distancia (pp. 68-102). Madrid: Ministerio de Trabajo y Asuntos Sociales.

Cobos, D. (2010). Una experiencia piloto de formación a distancia para delegados de prevención de riesgos laborales. Salud de los trabajadores, 18(2), 129-139.

Dean, P., Stahl, M. J., Sylwester, D. L., \& Peat, J. A. (2001). Effectiveness of combined delivery modalities for distance learning and resident learning. Quaterly Review of Distance Education, 2(3), 247-254.

Doxa (2005). E-Learning en las grandes empresas, panel anual. Resultados año 2005. Madrid: Grupo Doxa.

Fleiss, J. L. (1981). Statistical methods for rates and proportions. Nueva York: John Wiley \& Sons.

Gagnon, M. (2005). Ergonomic identification and biomechanical evaluation of workers' strategies and their validation in a training situation: summary of research. Clinical Biomechanics, 20(6), 569-580.

García Aretio, L. (1989). Para qué la educación a distancia. Madrid: UNED.

García del Dujo, A., \& Suárez, C. (2011). Interacción virtual y aprendizaje cooperativo. Un estudio cualitativo. Revista de Educación, 354, 473-498.

Gotsch, A. R., \& Weidner, B. L. (1994). Strategies for evaluating the effectiveness of training programs. Occupational Medicine, 9(2), 171-188.

Johnson, J. (2008). Improving safety training. Occupational Health E Safety, 77(11), 48, 50-1. 
Marcelo, C. (2011). E-Learning en la formación para el empleo: ¿qué opinan los usuarios? Revista de Educación, 355, 285-308.

Paulsson, K., Ivergard, T., \& Hunt, B. (2005). Learning at work: Competence development or competence-stress. Applied Ergonomics, 36(3), 135-144.

Robertson, M. M., Huang, Y. H., O’Neill, M. J., \& Schleifer, L. M. (2008). Flexible workspace design and ergonomics training: Impacts on the psychosocial work environment, musculoskeletal health, and work effectiveness among knowledge workers. Applied Ergonomics, 39(4), 482-494.

Roselli, N. (2010). Comparación experimental entre tres modalidades de enseñanza mediadas informáticamente. Revista de Investigación Educativa, 28(2), 265-282.

Ruiz Mafe, C., \& Tronch, J. (2007). La formación a distancia: caracterización y perspectivas de futuro. Capital Humano, 213, 76-83.

Sorine, A. J., Walls, R. T., \& Trinkleback, R. W. (2001). Safety training gets wired through web-based e-learning. Occupational Hazards, 63(2), 35-40.

Torres, J. J., \& Perera, V. H. (2009). Cálculo de la fiabilidad y concordancia entre investigadores de un sistema de categorías para el estudio del foro online en e-Learning. Revista de Investigación Educativa, 27(1), 89-103.

Wallen, E. S., \& Mulloy, K. B. (2006a). Computer-based training for safety: Comparing methods with older and younger workers. Journal of Safety Research, 37(5), 461-467.

Wallen, E. S., \& Mulloy, K. B. (2006b). Multimedia for occupational safety and health training: A pilot study examining a multimedia learning theory. Industrial Health, 44(4), 661-664.

Weidner, B. L., Delnevo, C., Gotsch, A. R., \& Rosen, M. (2001). The influence of trainee characteristics on the effectiveness of health and safety training for hazardous waste site operations work. Applied Occupational Environmental Hygiene, 16(11), 1049-1055.

Fecha de recepción: 21 de enero de 2013.

Fecha de revisión: 22 de enero de 2013.

Fecha de aceptación: 27 de junio de 2013. 